\title{
Produção de suspensões nanofibrilares de celulose vegetal por meio de processo combinado - Avaliação do gasto energético
}

\author{
Production of nanofibrillary suspensions of \\ vegetable cellulose by means of a combined \\ process - Energy expenditure evaluation
}

Débora Duarte Ribes ${ }^{1}$, Paula Zanatta ${ }^{1}$, Darci Alberto Gatto ${ }^{1}$, Washington Luiz Esteves Magalães ${ }^{2}$, Rafael Beltrame ${ }^{1}$

\footnotetext{
${ }^{1}$ Centro de desenvolvimento tecnológico, EIM/PPGCEM/UFPel, CEP: 96010-290, Pelotas, Rio Grande do Sul, Brasil.

${ }^{2}$ Empresa Brasileira de Pesquisa Agropecuária - Embrapa CEP: 83411-000, Colombo, Paraná, Brasil.

e-mail: deboraribes@hotmail.com, zanatta_paula@hotmail.com, beltrame.rafael@yahoo.com.br, darcigatto@pq.cnpq.br, Washington.magalhaes@embrapa.br
}

\section{RESUMO}

O presente estudo tem por objetivo produzir nanofibrilas de celulose vegetal com polpa Kraft marrom e branqueada por meio de um pré-tratamento enzimático e posteriormente um mecânico, denominado assim como processo combinado, com o intuito de diminuição de gasto energético. Para isso, as amostras de polpas de celulose foram previamente desestruturadas e encharcadas em água destilada com concentração de $3 \%$ de sólidos, afim de homogeneizá-las. Na hidrólise enzimática utilizou-se a enzima comercial Cellic CTec2, em porcentagens que variaram de 0,01 a $0,1 \%$ por diferentes períodos ( 1 e 2 horas). Após o tempo de hidrólise, a polpa foi passada em um moinho de discos, onde nesse processo ocorreu a medida do consumo do gasto energético com auxílio de um medidor de energia. Para a produção do gel, as polpas foram processadas pelo moinho de disco em ciclos de passagens. Para a inibição enzimática o conteúdo foi aquecido à $85^{\circ} \mathrm{C}$. Os géis foram armazenados em resfriamento de $5^{\circ} \mathrm{C}$. O processo foi caracterizado pelo gasto energético medido a cada amostra, além de ser medido o rendimento de cada gel produzido. De acordo com os resultados o processo combinado, apresentou-se viável para a produção de nanofibrilas de celulose vegetal, pois este diminui o gasto energético comparado com o processo mecânico e com a menor carga enzimática $(0,01 \%)$. A polpa marrom mesmo sem ter passado por processos de deslignificação mostrou-se promissora na produção das nanofibrilas de celulose vegetal.

Palavras-chave: gasto energético, processo combinado, polpa kraft, nanofibrilas de celulose, enzima Cellic CTec2

\begin{abstract}
The present study had as objective to produce vegetable cellulose nanofibrils with brown and bleached Kraft pulp by means of a pretreatment enzymatic and later a mechanical,known as combined process, with the purpose of decreased the energy waste. For this, cellulose pulp samples were previously destructured by water saturation and dismantled in a mechanical sheredder, after were stored under refrigeration at $5^{\circ} \mathrm{C}$. For the enzymatic pretreatment, it was used the commercial enzyme Cellic CTec2, in percentages ranging from 0.01 to $0.1 \%$ in a controlled hydrolysis with neutral $\mathrm{pH}$, at ambient temperature for one hour, being that the $0.01 \%$ amount samples underwent 1 and 2 hors enzymatic hydrolysis. After the time of hydrolysis, the pulp was passed in a disk mill, where in this process the consumption of the energy was measured with the aid of an energy meter. For the production of the gel, the pulps were processed by the disk mill passages cycles. For enzymatic inhibition the contents ewere heated to $85^{\circ} \mathrm{C}$. The gels were stored at $5^{\circ} \mathrm{C}$. The process was characterized by the measure energy expenditure for each sample and its controls, in addition to measuring the yield of each gel produced. According to the results the combined process was feasible for the production
\end{abstract}


of nanofibrils of vegetable cellulose, as this reduces the energy expenditure with the mechanical process and with the lowest enzymatic loading $(0.01 \%)$. The brown pulp, even without delignification processes, was shown to be promising in the production of plant cellulose nanofibrils.

Keywords: energetic waste; Combined process; Kraft pulp; celulose nanofibrils, Cellic CTec2

\section{INTRODUÇÃO}

A nanocelulose é considerada um nanomaterial, o qual tem se destacado recentemente por apresentar propriedades e comportamentos potencialmente superiores as fibras convencionais de celulose vegetal, além de ser sustentável, visto que é possível obtê-la de fontes renováveis [1,2].

As aplicações da nanocelulose são variadas e incluem agente de reforço em materiais compósitos, devido à alta razão de aspecto, baixa densidade e elevado módulo elástico [3,4], além de outras propriedades atraentes como, biocompatibilidade e biodegradabilidade, barreira para gás, absorção de água, propriedades reológicas e propriedades óticas [1]. Algumas aplicações são: embalagens, papéis opticamente transparentes para dispositivos eletrônicos, agente texturizante, curativos e implantes bioartificiais $[1,4]$.

As nanofibrilas de celulose (NFC) apresentam menor componente do que fibras de celulose, onde possui diâmetro na faixa de 2-20nm e comprimento até vários micrômetros, dependendo da sua origem [5]. Devido à abundância, menor dimensão e alta relação superfície-volume combinado com excelentes condições físicas e propriedades, as NFC estão sendo estudas para diversas aplicações em ciência dos materiais $[6,7,8]$.

As NFC podem ser extraídas da parede celular das plantas. Esta, constitui uma estrutura complexa em que as moléculas de celulose são intimamente associadas a outros polissacarídeos: lignina e hemicelulose. Esses elementos, em nanoescala, que compõe as fibras de celulose são ligados por fortes forças coesivas. Para desintegrar tais fibras se faz necessário um tratamento intenso, transformando, portanto, as fibras de celulose em nanofibrilas [9].

Os processos de obtenção típicos da nanocelulose podem ser classificados em top-down, no qual a nanosestrutura é alcançada, através de processos mecânicos, resultando em nanofibrilas, ou por meio de hidrólise ácida, para produção de nanocristais de celulose [10]. Em geral, as fontes dos processos top-down incluem madeira e fibras naturais, polpa de celulose, plantas e resíduos florestais. Outra fonte de produção é a biossíntese bacteriana, processo classificado como bottom-up, em que a nanoestrutra é organizada pela ação de bactérias em meios contendo açúcares e álcoois, resultando nas celuloses bacterianas [11,12].

Os dois processos mencionados, apresentam problemas relacionados à escala de produção tecnológica [13]. Os processos top-down mecânicos, possuem gasto energético muito elevado [14]. Vários métodos de fibrilação mecânica têm sido utilizados para a produção de NFC, tais como, homogeneizadores [15], microfluidizadores [16] e moedores [17]. Em combinação a estes, podem ser utilizados processos químicos ou processos enzimáticos [18].

A ação enzimática varia em função da composição da polpa a ser utilizada, devido a acessibilidade e degradabilidade da matriz fibrosa [19]. Em geral, os fatores que influenciam na hidrólise são: natureza da fibra (porosidade, área superficial específica e distribuição dos componentes químicos da fibra), quantidade de componentes químicos da fibra, conteúdo de lignina e estado da superfície das fibras [19, 20,21].

Portanto, uma compreensão na utilização de um pré-tratamento biológico pode vir a agregar nos processos de produção de nanofibrilas, visando produções ecologicamente corretas e de baixo custo, melhorando assim, a aceitação de um produto com alto valor agregado e com benefícios ainda inestimáveis.

Sendo assim, o objetivo deste estudo é produzir suspensões (géis) nanofibrilares de celulose vegetal a partir de um processo combinado, utilizando como pré-tratamento de enzimas celulases em diferentes aspectos de tempo e concentrações, visando a produção econômica e ecológica de nanofibrilas de celulose vegetal.

\section{MATERIAIS E MÉTODOS}

\subsection{Matéria-prima}

As amostras de partida utilizadas para o estudo foram de polpa kraft marrom e branqueada de eucalipto fornecidas pela empresa CMPC-Celulose Riograndense, situada na cidade de Guaíba - RS (Brasil). 
As enzimas comerciais denominadas Cellic CTec2 foram cedidas pela empresa Novozyes. Esta possui atividade sobre papel de filtro $\mathrm{n}^{\circ} 1\left(\mathrm{U}_{\mathrm{fp}}\right)$ de $583 \mathrm{~mL}^{-1}$ de extrato, sendo utilizada como referência para a atividade global das enzimas celulolíticas que agem sinergicamente na hidrólise de celulose [22].

\subsection{Preparo das fibras (polpa)}

As fibras de polpa kraft branqueada se encontravam em forma de folha de polpa seca, já as polpas kraft marrom se encontravam em agregados de polpa seca. Ambas primeiramente foram desagregadas e encharcadas a fim de homogeneizá-las, com uma concentração de 3\% de sólidos.

\subsection{Produção de NFC (processo combinado)}

Para a utilização das polpas na hidrólise enzimática, nas amostras homogeneizadas com concentração de 3\% foram adicionadas as concentrações de enzimas conforme a Tabela 1, destacando-se as amostras testemunhas, onde essas não sofreram o tratamento enzimático, a fim de comparação com as demais.

Tabela 1: Tratamento enzimático das nanofibrilas de celulose oriundas de polpa marrom e branqueada.

\begin{tabular}{|c|c|c|c|}
\hline TRATAMENTOS & CONCENTRAÇÃO (\%) & TEMPO & TEMPERATURA $\left(\mathbf{C}^{\circ}\right)$ \\
\hline PMT & 0,00 & Oh & \multirow{12}{*}{ T. ambiente $\left(24^{\circ} \mathrm{C}\right)$} \\
\hline$\overline{P M 1}$ & 0,01 & $1 \mathrm{~h}$ & \\
\hline PM2 & 0,01 & $2 \mathrm{~h}$ & \\
\hline PM3 & 0,02 & $1 \mathrm{~h}$ & \\
\hline$\overline{\text { PM4 }}$ & 0,05 & $1 \mathrm{~h}$ & \\
\hline PM5 & 0,1 & $1 \mathrm{~h}$ & \\
\hline$\overline{\mathrm{PBT}}$ & 0,00 & $0 \mathrm{~h}$ & \\
\hline$\overline{\mathrm{PB} 1}$ & 0,01 & $1 \mathrm{~h}$ & \\
\hline PB2 & 0,01 & $2 \mathrm{~h}$ & \\
\hline $\begin{array}{l}\text { PB3 } \\
\end{array}$ & 0,02 & $1 \mathrm{~h}$ & \\
\hline $\begin{array}{l}\mathrm{PB} 4 \\
\end{array}$ & 0,05 & $1 \mathrm{~h}$ & \\
\hline PB5 & 0,1 & $1 \mathrm{~h}$ & \\
\hline
\end{tabular}

*PMT: Gel marrom testemunha; PM: Gel marrom; PBT: Gel branco testemunha; PB: Gel branco

Para o processo mecânico foi utilizado um Microprocessador Super MASSCOLLOIDER MASUKO SANGYO em 1.500rpm. A polpa foi alimentada continuamente para moinho coloidal, que é constituído de dois discos de moagem de pedra colocados no topo um do outro. Este foi operado na moagem de contato com o intervalo dos dois discos ajustados. A presença da polpa entre os discos assegura que não exista nenhum contato direto entre os discos. No momento que as amostras de fibrilas ficam com um aspecto de gel viscoso, onde não consegue-se observar mais fibras no gel o processo é interrompido. Para a inibição enzimática os géis são aquecidos a uma temperatura de $85^{\circ} \mathrm{C}$ e posteriormente armazenados em resfriamento de $5^{\circ} \mathrm{C}$.

À energia consumida pelo processo mecânico foi registrado usando um medidor de energia (Mult - da KRON) ligado ao moinho coloidal.

\subsection{Rendimento de NFC}

$\mathrm{O}$ rendimento fornece informação relativa à quantidade de celulose inicial que foi nanofibrilada. Para isso, foi realizada a centrifugação, o material decantado após a centrifugação foi seco a $105^{\circ} \mathrm{C}$ até peso constante. Após a secagem, aplicou-se a Equação 1.

$$
\eta(\%)=\left(\frac{1-\text { massa se dimentada } \sec a}{\text { massa inicial de amostra } x \% \text { solidos }}\right) \times 100
$$




\subsection{Propriedades Reológicas - Viscosidade}

As propriedades reológicas foram obtidas em um viscosímetro Brookfield modelo RVDV-II+PRO, que determina as propriedades reológicas de líquidos Newtonianos e Não Newtonianos. As análises foram determinadas em temperatura de $24^{\circ} \mathrm{C}$ com fuso de SC4-21.

Dentre as formas de análises que podem ser feitas com os dados coletados, optou-se por obter a curva de tensão de cisalhamento $\mathrm{x}$ taxa de cisalhamento (curva de fluxo), das quais tornou-se possível fazer uma análise comparativa entre as amostras dos comportamentos dependentes do tempo.

\subsection{Consumo energético}

O controle do gasto de energia foi determinado através de um medidor de energia do tipo Mult - K (KRON). Este foi acoplado ao moinho, onde todas as amostras foram controladas durante o tempo de fibrilação. O medidor foi estabelecido no modo de Energia (Ene), onde a grandeza que foi verificada foi a de consumo de energia ativa positiva, tendo o seu valor em unidades de KWh.

\subsection{Análise estatística}

Os dados obtidos foram processados pelo programa estatística Statgraphics, onde se realizou a análise de variância com (ANOVA) 95\% de confiança com teste de média de Tukey.

\section{RESULTADOS E DISCUSSÃO}

\subsection{Rendimento de nanofibrilas de celulose (NFC)}

O rendimento da nanofibrilação depende do número de passagens da celulose e da pressão utilizadas no moinho. O objetivo de utilizar o pré-tratamento enzimático é formar uma suspensão estável de nanofibrilas de celulose com uma diminuição de passagens pelo moinho, para as polpas marrom (PM) e branqueada (PB), obtendo assim um maior rendimento de sólidos, os quais representam a maior quantidade de nanofibrilas presentes nas suspensões produzidas.

Na Tabela 2 pode-se observar que o gel marrom (PM) obteve um rendimento mais elevado do que a suspensão de gel branco (PB). Isto ocorre devido ao processo de branqueamento Kraft das polpas bancas, onde essas perdem uma porcentagem de hemicelulose, sendo essa uma grande fonte de fibras de uma amostra vegetal.

Dentro do processo de obtenção de cada suspeção, obteve-se diferença significativa entre todos os tratamentos comparando-os com suas testemunha. ANDERSEM et. al. [19] revelam que a quantidade de lignina presente nas fibras pode interferir no rendimento das suspensões. Essa explicação comprova com o fato da PM ter obtido maior rendimento, pois a quantidade de enzimas afeta o rendimento pois tem capacidadede degradade a lignina e as partes amorfas das fibrilas [19,21].

Observar-se que para as suspensões marrom, a amostra que obteve maior rendimento foi a amostra PM2 seguida pela PM5, esse fenômeno ocorre devido a amostra PM2 ter sofrido o maior tempo de hidrolise e PM5 a maior carga enzimática utilizada, onde ambas são responsáveis pela maior desfibrilação das amostras.

Essa afirmação pode ser explicada pela capacidade de enzimas celulases degradar os componentes lignocelulosicos, por tanto as amostras de polpa marrom por possuírem uma quantidade de lignina onde essa possui cadeias químicas amorfas, facilitando a sua degradação [24].

As amostras com polpas branqueadas que sofreram tratamento de hidrólise enzimática obtiveram diferença significativas comparadas com sua testemunha, destacando a amostra PB5, a qual obteve maior rendimento do que a testemunha, diferentemente das demais amostras. 
Tabela 2: Média de rendimento das suspensões nanofibrilas.

\begin{tabular}{lll}
\hline & Amostra & Média Rendimento \% \\
\hline & PMT & $99,39 \mathrm{a}$ \\
& PM2 & $98,98 \mathrm{~b}$ \\
& PM5 & $98,48 \mathrm{c}$ \\
& PM4 & $98,38 \mathrm{~d}$ \\
& PM1 & $98,36 \mathrm{~d}$ \\
& PM3 & $98,33 \mathrm{~d}$ \\
\hline & Valor $-\mathrm{F}$ & 320,77 \\
& Valor - P & 0 \\
\hline & PB5 & $98,45 \mathrm{a}$ \\
& PB3 & $98,18 \mathrm{~b}$ \\
& PBT & $98,13 \mathrm{c}$ \\
PB4 & $97,98 \mathrm{~d}$ \\
PB1 & $97,68 \mathrm{e}$ \\
Valor $-\mathrm{F}$ & $97,05 \mathrm{f}$ \\
\hline
\end{tabular}

Onde: PMT: gel marrom testemunha; PM: gel marrom; PBT: gel branco testemunha; PB: gel branco; média na coluna seguidas por letras diferentes apresentam diferença estatisticamente significativa, de acordo com o teste de média de tukey; $\mathrm{F}=$ razão entre o estimado dentro de um tratamento e diferentes tratamentos; $\mathrm{p}=$ valor relacionado a razão $\mathrm{F}$, sendo que acima de 0,05 não apresenta diferenças significativas.

De acordo com LIMA e BORSALI [20], as variáveis que podem afetar o processo do comportamento das enzimas sobre as fibras são: consistência da polpa, grau de agitação e pH. Sendo que a ação enzimática varia em função da composição da polpa a ser utilizada, podendo assim explicar a diferença de rendimento entre as polpas brancas e marrons.

Pode-se dizer que uma das possibilidades pode ser a diferença de acessibilidade e degradabilidade da matriz fibrosa. Em geral, os fatores que vão interferir na hidrólise enzimática são: natureza da fibra, quantidade de componentes químicos da fibra; conteúdo de lignina e estado da superfície das fibras [19,21]. Assim, essa afirmação se aplica a polpa marrom, pois a mesma, não passou por uma etapa de branqueamento, onde a composição química permanece rica, onde há maior quantidade de lignina presente na polpa, assim como os demais componentes, celulose, hemicelulose e extrativos [23].

$\mathrm{Na}$ Figura 1 são apresentadas as curvas de fluxo (tensão de cisalhamento x taxa de cisalhamento) das amostras de nanofibrilas de celulose obtidas através do método combinado, a partir de polpa kraf marrom e branqueada. 

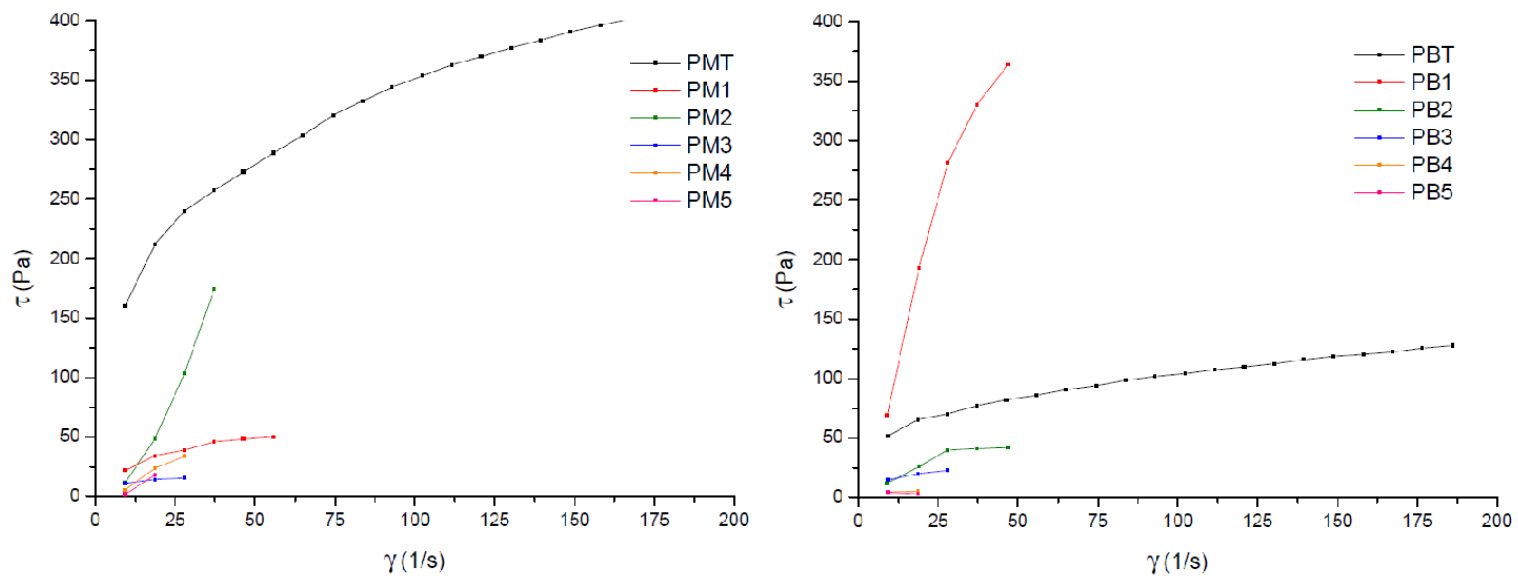

Figura 1: Curvas de fluxo (tensão de cisalhamento x taxa de cisalhamento). Onde: PMT: gel marrom testemunha; PM: gel marrom; PBT: gel branco testemunha: PB: gel branco.

Verificou-se um comportamento levemente pseudoplástico, através da curvatura, mostrando as diferenças de comportamentos tixotrópicos. Para a amostra de polpa marrom, observa-se que a taxa de cisalhamento diminuiu consideravelmente. [25] relatam que a taxa de cisalhamento é particularmente interligada a cristalinidade da suspensão.

Conforme relata TOBIAS et. al. [22], para partículas finas $(<10 \mu \mathrm{m})$, as iterações químicas da superfície tornam-se significativas, facilitando a agregação. Para maiores porcentagens de sólidos, essas partículas se interconectam, formando uma estrutura de rede, com uma tensão limite de escoamento associada. Se o cisalhamento quebra essa estrutura, o comportamento será pseudoplástico com tensão limite de escoamento e tixotrópico.

Contudo as amostras que obtiveram menor carga enzimática o comportamento mudou, obtendo um comportamento reológico diferenciado em relação as demais, através do aumento das inclinações das curvas de fluxo da histerese que é muito maior. Isto indica que estão sofrendo processo de gelificação com as taxas de cisalhamento aplicadas.

Segundo TOBIAS et. al. [22], alta porcentagem de sólidos nas suspensões proporciona um comportamento pseudoplástico. WANG et. al. [26] relata que o valor da fração de sólidos máxima depende da distribuição de tamanho de partículas e tipicamente varia de 61 a $70 \%$ de sólidos em volume. Conforme foi observado na Tabela 2, o aumento de rendimento com o aumento da carga enzimática para a PB, podendo afirmar assim que ocorreu o aumento do teor de sólidos.

A Tabela 3 apresenta os dados de consumo de energia e tempo no preparo das suspensões marrom e branco de nanofibrilas de celulose pelo processo combinado e a Figura 2 mostra o tempo em relação ao gasto energético.

Cabe ressaltar que para a variável consumo de energia não se realizou análises estatísticas, pois não houve repetições para a PM e PB em razão do elevado custo da enzima utilizada. Com isso, realizou-se apenas uma análise por amostra para o consumo energético.

Estatisticamente a polpa branca obteve um maior redutor do consumo de energia comparada à polpa original sem tratamento, sendo esse de $45 \%$, já a polpa marrom obteve uma redução de $33 \%$.

Tabela 3: Consumo energético, tempo de preparo e rendimento dos géis de nanofibrilas referentes ao tratamento biotecnológico.

\begin{tabular}{lllll}
\hline TRATAMENTOS & CONSUMO (KW.H) & TEMPO (H) & RENDIMENTO (\%) & $\begin{array}{l}\text { CONCENTRAÇÃO DE } \\
\text { SÓLIDOS (\%) }\end{array}$ \\
\hline PMT & & & 0,57 \\
PM1 & 0,209 & 0,516 & 99,39 & 1,44 \\
PM2 & 0,163 & 0,400 & 98,37 & 0,94 \\
PM3 & 0,122 & 0,366 & 98,99 & 1,47 \\
\hline
\end{tabular}




\begin{tabular}{lllll}
\hline PM4 & 0,100 & 0,300 & 98,38 & 1,52 \\
PM5 & 0,069 & 0,233 & 98,49 & 1,35 \\
PBT & 0,216 & 9,583 & 98,19 & 1,59 \\
PB1 & 0,483 & 97,06 & 2,37 \\
PB2 & 0,182 & 97,98 & 1,73 \\
PB3 & 0,155 & 0,366 & 98,14 & 1,62 \\
PB4 & 0,161 & 0,333 & 97,69 & 1,97 \\
PB5 & 0,116 & 0,283 & 98,46 & 1,39
\end{tabular}

Onde: * PMT: gel marrom testemunha; PM: gel marrom; PBT: gel branco testemunha: PB: gel branco.

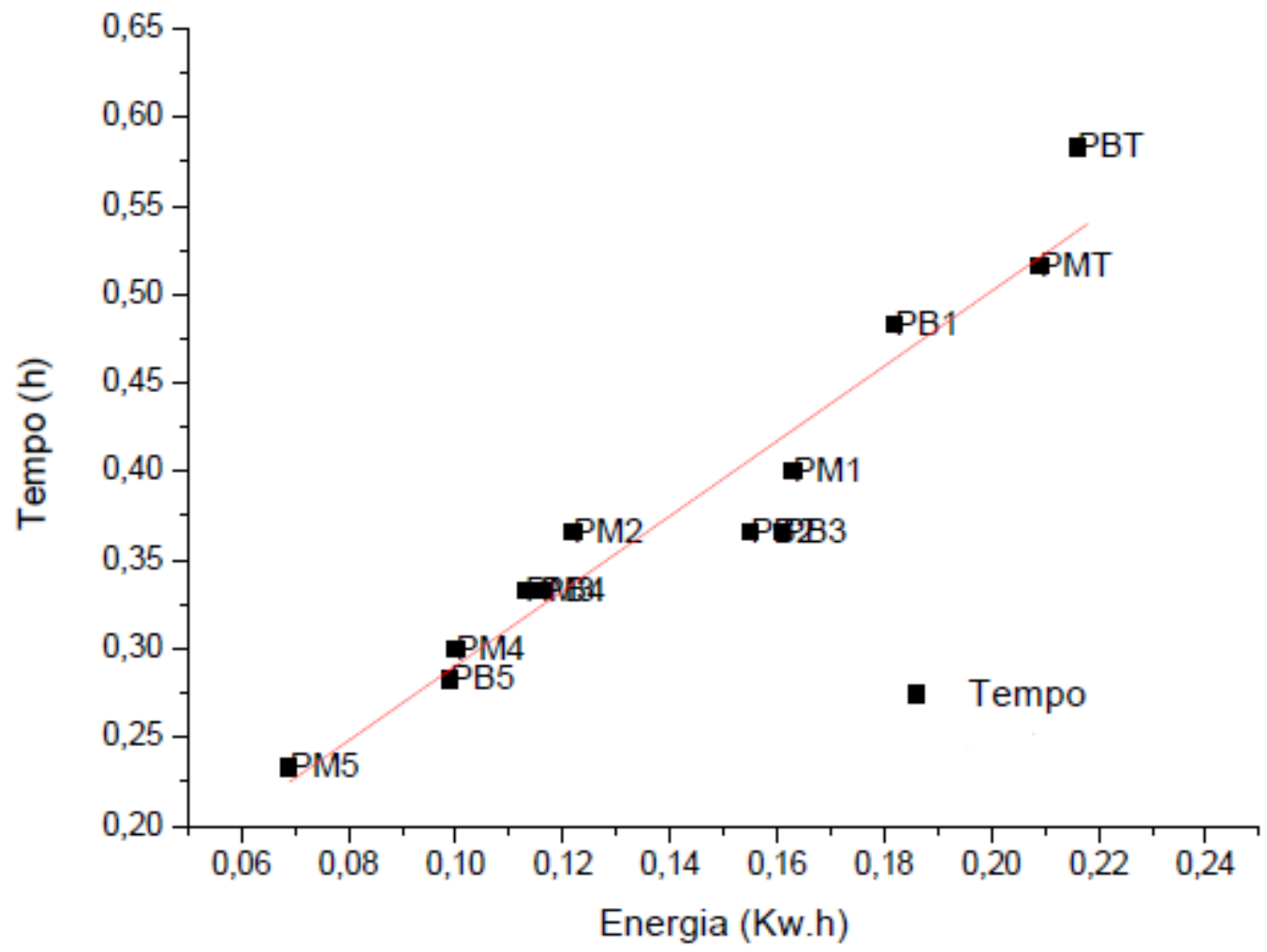

Figura 2: Consumo de tempo em relação ao gasto de energia. Onde: PMT: gel marrom testemunha; PM: gel marrom; PBT: gel branco testemunha: PB: gel branco.

Na Figura 2, consegue-se observar que o tempo se encontra em forma linear com o consumo da energia, sendo assim pode-se dizer que o moinho consome a mesma energia e que o tempo de processamento diminui para polpas mais fáceis de desfibrilar, como é o caso das amostras com maior carga enzimática e maior tempo de hidrolise.

Segundo KEREKES [27], a energia especifica e a intensidade do micro moedor pode ser muito elevada, por esse motivo quanto mais homogênea, a amostra sofre mais impacto, portanto diminui o gasto energético. Isto demonsta que o processo combinado diminui consideravelmente o gasto energético com o aumento do rendimento da suspensão obtida para ambas polpas.

\section{CONCLUSÕES}

Com o estudo realizado conclui-se que o processo combinado é eficiente para produção de suspensões nanofibriladas de celulose. Diferenciando os tipos de polpas utilizadas para esta metodologia, as amostras 
obtidas através de polpa marrom demonstraram ser mais viável na produção de nanofibrilas de celulose, sendo a utilização de menor carga enzimática com duas horas de hidrólise um tratamento suficiente para gerar uma suspensão de celulose com alto rendimento.

\section{AGRADECIMENTOS}

Os autores gostariam de agradecer a Embrapa-Florestas, a empresa Novozyes e ao Conselho Nacional de Desenvolvimento Científico e Tecnológico $(\mathrm{CNPq})$ e a pelo suporte financeiro.

\section{BIBLIOGRAFIA}

[1] KLEMM, D., KRAMER, F., MORITZ, S., et al., "Nanocelluloses: a new family of nature-based materials", Angewandte Chemie (International ed. in English), Weinheim, DE, v. 50, n. 24, pp. 5438-5466, 2011.

[2] PETERSSON, L., KVIEN, I., OKSMAN, K., "Structure and termal properties of poly (lactic acid)/ cellulose whiskers nanocomposites materials", Composite Science and Technology, v.67, n.11-12, pp. 253525442007.

[3] SIQUEIRA G., DUFRESNE A. "Cellulose Whiskers versus Microfibrils: Influence of the nature of the nanoparticle and its surface functionalization on the thermal and mechanical properties of nanocomposites", Biomacromolecules, v.10, pp. 425-432, 2010.

[4] EICHHORN, S.J., BAILLIE, C.A., ZAFEIROPOULOS, N., et al., Review - "Current international research into cellulosic fibres and composites”, Journal of Materials Science, v.36, pp. 2107-2131, 2009.

[5] STELTE, W., SANADI, A. R. "Preparation and Characterization of Celulose Nanofibers from Twow Commercial Hardwood and Softwood Pulps", Industrial \& Engineering Chemistry Research, v. 48, n. 24, pp. 11211-11219, 2009.

[6] OUAJAI, S., SHANKS, R. "Composition, structure and thermal degradation of hemp cellulose after chemical treatments”, Polymer Degradation and Stability, v. 89, n. 2, pp. 327-335, 2005.

[7] KAUSHIK A, SINGH M. "Isolation and characterization of cellulose nanofibrils from wheat straw using steam explosioncoupled with high shear homogenization”, Carbohydr Res., v. 346, pp.76-85, 2011.

[8] HE, M. Slurry rheology of industrial minerals and its effects on wet ultra-fine grinding, Ph.D Thesis, Sweden, Luleå University of Technology, 2012.

[9] UETANI, K., YANO, H. "Nanofibrilation of wood pulp using a high-speed blender", Biomacromolecules, Washington, v. 12, n. 2, pp. 348-353, 2011.

[10] NAKAGAITO, A. N., YANO, H. "The effect of fiber content of mechanical and thermal expansion properties of biocomposites based on micro fibrillated celulose”, Cellulose, v.15, n. 6, pp.555- 559, 2008.

[11] ABDUL KHALIL, H., BHAT, A., IREANA YUSRA, A. "Green composites from sustainable cellulose nanofibrils: a review”, Carbohydrate Polymers, v. 87, n. 2, pp. 963-979, 2012.

[12] MANDAL, A., CHAKRABARTY, D. "Studies on the mechanical, thermal, morphological and barrier properties of nanocomposites based on poly(vinyl alcohol) and nanocellulose from sugarcane bagasse". Journal of Industrial and Engineering Chemistry, v. 20, n. 2, p. 462-473, 2014.

[13] SIXTA, H. Handbook of pulp, Wiley-VCH. Weinheim, 2006.

[14] SIRÓ, I., PLACKETT, D., HEDENQVIST , M.S., et al. "Highly transparente Films carboxymethylated microfibrillated celulose: the effect of multiple homogenzation steps o key properties", Journal of Applied Polymer Science, New York, v. 119, n. 5, pp. 2652-2660, 2011.

[15] CHINGA-CARRASCO, G. "Cellulose fibres, nanofibrils and microfibrils: Themorphological sequence of MFC components from a plant physiology and fibretechnology point of view", Nanoscale Research Letter. v. 6, n. 1, 417, 2012.

[16] DUFRESNE, A. "Interfacial phenomena in nanocompósitos based on polysaccharide nanocrystals", Composite. Interfac, v. 10, n. 4-5, 2003.

[17] ABE, K., YANO, H. "Comparison of the characteristics od celulose microfibril aggregates of wood, ric straw and potato tuber", Cellulose, v. 16, n. 6, pp. 1017-1023, 2007.

[18] DASH, R, FOSTON, M, RAGAUSKAS, A.J. "Improving the mechanical and thermal properties of 
gelatin hydrogels cross linked by cellulose nanowhiskers”, Carbohydr Polym , v.91, n.2, pp. 638-645, 2013. [19] ANDRESEN, M., STENSTAD, P., MORETRO, T., "Nonleaching antimicrobial films prepared from surfasse-modfiede microfibrilated celulose", Biomacromoecules, v.8, n.7, pp. 2149-2155, 2007.

[20] SOUZA LIMA, M.M., BORSALI, R. "Rodlike Cellulose Microcrystals: Structure, Properties, and Applications", Macromol. Rapid Commun. v.25, pp.771-787, 2002.

[21] EICHHORN, S. J., et al., "Review: current international research into cellulose nanofibres and nanocomposites", Journal of Materials Science, v. 45, n. 1, p. 1-33, 24 set. 2010.

[22] TOBIAS, M., KARIN, S., GUNNAR, W., et al., "Rheological properties of nanocellulose suspensions: effects of fibril/particle dimensions and surface characteristics", Cellulose, v.24, n.6, pp. 2499-2510, 2017.

[23] MEYABADI, T. F., DADASHIAN, F. "Optimization of Enzymatic Hydrolysis of Waste Cotton Fibers for Nanoparticles Production Using Response Surface Methodology”, Fibers and Polymers, v. 13, n. 3, pp. 313-321, 2012.

[24] MORAIS, J. P. S., ROSA, M. D. F., NASCIMENTO, L. D., et al., "Extraction and characterization of nanocellulose structures from raw cotton linter", Carbohydrate Polymers, v. 91, n. 1, p. 229-235, 2013.

[25] MC CABE, W. L., SMITH, J. C., HARRIOUT, P. Unit Operations of Chemical Engineering. 7a. New York, 2005.

[26] WANG, S., ZHANG, X., HU, L., et al., "Controlling the structure and rheology of TEMPO-oxidized cellulose in zinc chloride aqueous suspensions for fabricating advanced nanopaper", Physical Review Journals 2016.

[27] KEREKES, R.J. “Characterizing refining action in PFI mills”, TAPPI, v.4, n.3, pp. 9-14, 2005. 